\title{
CFD Analysis of Heat Transfer Enhancement in Pipe Flow using Nanofluid
}

\author{
Pankaj Ghatage ${ }^{1}$, Abhijeet Shah $^{2}$, Durgesh Kumar Chavan ${ }^{3}$ \\ Assistant Professor, Automobile Engineering Department, R.I.T., Islampur, India ${ }^{1,2}$ \\ Head, Diploma Mechanical, Govt. Polytechnic, Ambad, India ${ }^{3}$
}

\begin{abstract}
With the demand for energy continues to grow globally, there is a need to make heat transfer equipment more energy efficient. At one hand, the exponential growth of electronics, communication and computer technology and their choice to go for miniaturization has put added pressure on the designer to create efficient thermal management devices for these systems. Particularly thermal conductivity of a fluid plays a vital role in the development of energy-efficient heat transfer equipment. An innovative idea is to suspend nano-sized solid particles in the fluid to improve heat transfer characteristics of the fluid. Nanofluids are the suspension of nanometer-sized particles in base fluids such as water and ethylene glycol. In convective heat transfer, number of other factors along with thermal conductivity affects heat transfer performance, so careful examination of convective heat transfer using nanofluid is required. For optimum condition of nanofluids, some experimentation is required for better result. As experimentation is time consuming process, nanofluids are analyzed by using CFD (computational fluid dynamics) approach. This approach has attracted the attention of researchers in the past decade, though the mechanism is not fully understood yet. So in this work, a systematic computational fluid dynamic investigation of $\mathrm{Al}_{2} \mathrm{O}_{3} /$ water nanofluid with different volume fraction by varying Reynolds number and constant wall temperature boundary condition has been carried out adopting single phase and two phase approach and the results are compared with experimental results and theoretical correlations. Both the single phase and two phase models results show a good agreement with experimental data and theoretical correlations but two phase model gives prediction of heat transfer rate compared to the single phase model.
\end{abstract}

Keywords: Nanofluid, convective heat transfer, $\mathrm{CFD}, \mathrm{Al}_{2} \mathrm{O}_{3}$ nanoparticles, Reynolds number.

\section{INTRODUCTION}

Heating or cooling fluids such as water, engine oil and ethylene glycol plays a crucial role in thermal management of high-tech industries but they have poor thermal characteristics, in particular thermal conductivity. Despite considerable efforts are made to improve the rate of heat transfer by usage of extended surfaces, minichannels and micro-channels, further enhancement in heating and cooling rate is always in demand. Since solid materials possess higher thermal conductivities, many studies have been carried out on thermal properties of suspension of solid particles in conventional heat transfer fluids.

With ever increasing thermal loads due to high power output and smaller features of microelectronics devices, improvement to make heat transfer equipment more energy efficient become most important technical issue. The thermal management of many applications like transportation, manufacturing and micro-electronics is very important, to maintain their desired performance and durability. The improvement to make heat transfer equipment more energy efficient can be made by focus on reducing the size on one hand and huge increase in heat flux on the other. Heat transfer fluids such as water, mineral oil and ethylene glycol are used in many industrial processes. The poor heat transfer properties of these common fluids compared to most solids is a primary obstacle to the high compactness and effectiveness of heat exchangers. As the thermal conductivity of a fluid plays a vital role in the development of energy efficient heat transfer equipment, there is need to find new heat transfer fluid which has higher thermal properties and an advanced cooling techniques. It can be obtained by suspending small particles of solid whose thermal conductivity is higher as compared to conventional fluids. It is well known that at room temperature, metallic solids posses the order of magnitude higher thermal conductivity than fluid. For example, the thermal conductivity of copper at room temperature is about 700 times greater than that of water and about 3000 times greater than that of engine oil. Therefore, the thermal conductivities of fluids containing solid metallic or nonmetallic (metallic oxide) particles would be expected to be significantly higher than those of conventional heat transfer fluids. If these particles are of millimeter or micrometer size, problems such as rapid settling of particles, clogging of flow channels and increased pressure drop may arises. If the fluid is kept circulating rapidly enough to prevent much settling, the micro particles would damage the walls of heat transfer equipment and wear out them.

To overcome above problems, new class of heat transfer fluid called nanofluid can be used. Nanofluid is heat transfer fluid in which nanometer sized solid particles are 
Vol. 4, Issue 1, January 2017

dispersed in traditional heat transfer fluid. These fluids exhibit significantly higher thermal properties, in particular, thermal conductivity, than those base fluids. Nanoparticles, due to their high surface to volume ratio can remain in suspension and thereby reduce erosion and clogging.

HaghshenasFard et al. [1] have studied laminar convective heat transfer of nanofluid in circular tube under constant wall temperature condition by using CFD approach and compared two phase and single phase models for prediction of laminar heat transfer in tube with constant wall temperature for $\mathrm{Cu}$ /water nanofluid with $0.2 \%$ volume concentration, the average relative error between experimental data and CFD results based on single phase model was $16 \%$ while for two phase model was $8 \%$.

Akbari et al. [2] have done systematic comparison of prediction for a laminar mixed convection flow which includes hydrodynamic characteristics and the effect of temperature dependent properties. The two phase models have given closer predictions of convective heat transfer coefficient to the experimental data than single phase model. Single and two phase models were found to be predicting identical hydrodynamic fields but very different thermal ones.

Moraveji et al. [3] have investigated convective heat transfer effect on the nanofluid flow in the developing region of a tube with constant heat flux by using computational fluid dynamics (CFD). Effect of particle size on convective heat transfer coefficient was investigated in Reynolds numbers range 500 - 2500 for various axial locations of tube. The results show that the heat transfer coefficient enhanced with increasing the nanoparticle concentration and Reynolds number. Further, the heat transfer coefficient decreased with increasing the axial location and particle diameter.

Perumal Kumar and Ganesan [4]have studied the heat transfer enhancement using aluminium oxide nanofluid using low and high volume fraction nanofluids in turbulent pipe flow with constant wall temperature by using Computational Fluid Dynamic approach (CFD) with single phase. A volume fraction of $1 \%$ is found to be an effective heat transfer enhancement technique. Both the experimental values and the numerical predictions show that heat transfer enhancement increases as the volume fraction of the nanoparticles increases. The single phase model predicts convective heat transfer coefficient with reasonable accuracy and considered good enough to test new nanofluids as it is computationally inexpensive compared to the multiphase approach.
P.Kumar [5] has studied the heat transfer enhancement using aluminium oxide nanofluid by computational fluid dynamic modeling, adopting the single phase approach. Both the experimental values and the numerical predictions showed that heat transfer enhancement in the laminar regime were not as significant as in the turbulent regime. Model predictions in the turbulent regime agree very well with experimental values but single phase approach does not predict heat transfer coefficient as accurately as in the turbulent regime, so that more research needs to be done to arrive at a definitive conclusion on the efficacy of single phase approach for laminar flow heat transfer prediction.

Pendalwar et al. [6]have studied and presented numerical and experimental investigation on convective heat transfer coefficient for turbulent flow using $\mathrm{Al}_{2} \mathrm{O}_{3}$-water nanofluid. Both experimental and numerical investigations show increase in heat transfer coefficient and Nusselt number with increase in nanoparticle volume fraction.

The nanofluid attracted great interest from the research community due to their potential benefits and applications in numerous important fields such as transportation, microelectronics, manufacturing, and medical etc. For optimum condition of nanofluids, some experimentation is required for better result. As experimentation is time consuming process, nanofluids are analyzed by using CFD (computational fluid dynamics) approach. This approach has attracted the attention of researchers in the past decade, though the mechanism is not fully understood yet. So in this work, a systematic computational fluid dynamic investigation has been carried out adopting single phase and two-phase approach and results are compared with experimental results.

\section{SYNTHESIS OF NANOFLUID}

In this study, $20 \mathrm{~nm} \gamma-\mathrm{Al}_{2} \mathrm{O}_{3}$ nanoparticles were used. The thermo physical properties which are shown in Table 1.The different nanofluids were prepared by mixing $\mathrm{Al}_{2} \mathrm{O}_{3}$ nanoparticles with different volume fraction in deionized water as a base fluid. The particles were initially mixed in base fluid using mechanical stirrer for two hours and then ultrasonicated for several hours to even and stable suspension of nanoparticles. In this study no dispersant or stabilizer was added as they may affect fluid properties. Even after ultrasonication, sedimentation of particles was observed, so stirrer was used during the experiment to minimize the sedimentation of the particles. Prepared nanofluid then used to investigate convective heat transfer coefficient using nanofluid.

TABLE I Physical properties of nanosized particles

\begin{tabular}{|c|c|c|c|c|}
\hline $\begin{array}{l}\text { Nanosized } \\
\text { particles }\end{array}$ & Mean Diameter (nm) & Density (Kg/m3) & $\begin{array}{l}\text { Thermal Conductivity } \\
(\mathbf{W} / \mathbf{m ~ K})\end{array}$ & $\begin{array}{l}\text { Specific Heat } \\
(\mathbf{J} / \mathbf{k g ~ K})\end{array}$ \\
\hline $\mathrm{Al}_{2} \mathrm{O}_{3}$ & 20 & 3700 & 46 & 880 \\
\hline
\end{tabular}


Vol. 4, Issue 1, January 2017

\section{III.EXPERIMENTAL SET UP}

\section{CFD MODELLING}

In order to study convective heat transfer under constant 4.1 Single and Two Phase Analysis

wall temperature boundary condition, an experimental set- The CFD approach uses a numerical technique for solving up is shown in fig. No.1.The experimental set-up consisted the governing equations for a given flow geometry and of flow loop containing several sections such as boundary conditions. In this study flow pattern, temperature, pressure and flow rate measuring unit heating temperature distribution and heat transfer coefficient and cooling section and flow controlling system. A 10 through a circular pipe was simulated using a commercial litter vessel equipped by drain valve was used as a CFD package, FLUENT 13.0. The use of CFD reduces the reservoir. In order to control the fluid flow rate, a reflux number of necessary experiments and gives results, which line valve was used. Test section consists of $1020 \mathrm{~mm}$ would hardly be accessible by measurements annular copper tube having inner diameter $10.6 \mathrm{~mm}$ and mathematical modelling of nanofluid consists of two thickness $0.85 \mathrm{~mm}$ with $150 \times 150 \mathrm{~mm}$ rectangular outer approaches namely single phase model and two phase stainless steel tube. A nanofluid flows inside the inner tube while heated water entered annular section which created constant wall boundary condition. The outer rectangular tube was insulated by fibre glass. In order to measure wall temperature five ( $\mathrm{k}$ type) thermocouples were welded on inner tube wall at equally spaced distance. Two (k type) thermocouples were welded at inlet and outlet of test section for measuring bulk temperature of nanofluid. The fluid leaves the test section passes through heat exchanger in which water is used as cooling fluid, and then flow measuring section. Flow was measured with flow meter. After filling the nanofluid with specified concentration in reservoir tank, the heater is started to heat the water and heated water is circulated to increase the tube wall temperature. Then pump and cooling system was started. After 80-90 min. the system reached steady state condition. The flow rate is regulated by using the valve on reflex line and tests were repeated at least five flow rates for each concentration. During experimental runs, inlet and outlet temperature of the nanofluid, the tube wall temperature different location and flow rate was measured.

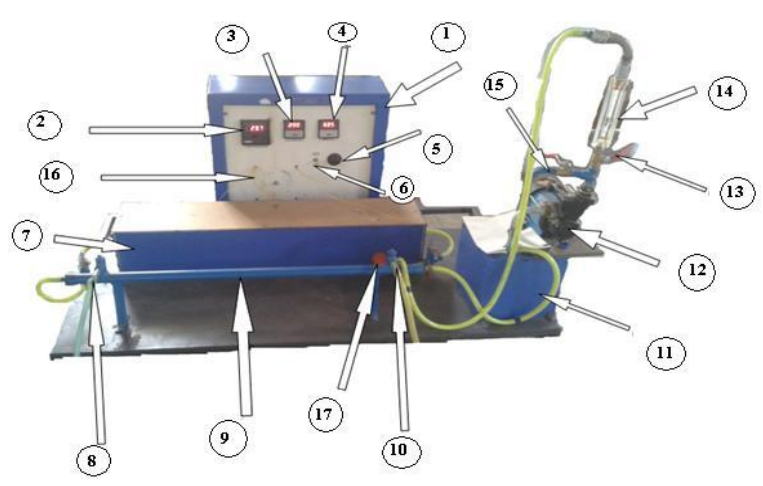

Fig.1 Experimental Set up

1. Control Panel

3. Voltmeter

5. Temperature Regulator

7. Heat Exchanger With

Test Section

9. Condenser

11. Reservoir

13. Valve

15. Bypass

17. Heater
2. Temperature Indicator

4. Ammeter

6. On-Off Switch

8. Condenser Inlet

10. Condenser Outlet

12. $0.5 \mathrm{Hp}$ Single Phase Pump

14. Flow meter

16. Thermocouples model. Nanofluid treated as single phase fluid and all motion and energy equations for pure fluid can be directly used to nanofluid. In two phase model, nanoparticles and base fluid were considered as two different phases with different momentum. To analyse two phase model, discrete phase modelling (DPM) technique is used. The detailed flow field for the single-phase and two-phase flows in a circular tube with constant wall temperature can be determined by solving the volume-averaged fluid equations, as follows;

4.1.1 Single-phase flow equations:-

Continuity equation

$$
\frac{\partial \rho}{\partial \mathrm{t}}+\nabla(\rho \mathrm{U})=0
$$

- Momentum equation:

$$
\frac{\partial}{\partial \mathrm{t}}(\rho \mathrm{U})+\nabla(\rho \mathrm{UU})=-\nabla \mathrm{P}+\nabla \tau+\mathrm{B}
$$

- Energy equation:

$$
\frac{\partial}{\partial t}(\rho h)+\nabla\left(\rho U C_{p} T\right)=\nabla(k \nabla T)
$$

4.1.2 Two-phase flow equations:-

- Continuity equation:

$$
\frac{\partial}{\partial \mathrm{t}}\left(\varphi_{\alpha} \rho_{\alpha}\right)+\nabla\left(\varphi_{\alpha} \rho_{\alpha} \mathrm{U}_{\alpha}-\mathrm{T}_{\alpha} \nabla \varphi_{\alpha}\right)=0
$$

- Momentum equation:

$$
\begin{gathered}
\frac{\partial}{\partial \mathrm{t}}\left(\varphi_{\alpha} \rho_{\alpha} \mathrm{U}_{\alpha}\right)+\left\{\varphi_{\alpha}\left[\rho_{\alpha} \mathrm{U}_{\alpha} \mathrm{U}_{\alpha}-\mu_{\mathrm{e} \alpha}\left(\nabla \mathrm{U}_{\alpha}+\left(\nabla \mathrm{U}_{\alpha}\right)^{\mathrm{T}}\right)\right]\right\} \\
=\varphi_{\alpha}\left(\mathrm{B}_{\alpha}-\nabla \mathrm{P}\right)+\mathrm{F}_{\alpha}
\end{gathered}
$$

- Energy equation:

$$
\begin{array}{r}
\frac{\partial}{\partial \mathrm{t}}\left(\varphi_{\alpha} \rho_{\alpha} \mathrm{h}_{\alpha}\right)+\nabla\left[\varphi_{\alpha}\left(\rho_{\alpha} \mathrm{U}_{\alpha} \mathrm{h}_{\alpha}-\mathrm{k}_{\alpha} \nabla \mathrm{T}_{\alpha}\right)\right] \\
=\sum_{\beta=1}^{\mathrm{N}}\left(\mathrm{T}_{\alpha \beta} \mathrm{h}_{\beta \mathrm{s}}-\mathrm{T}_{\beta \alpha} \mathrm{h}_{\alpha \mathrm{s}}\right)+\mathrm{Q}_{\alpha}+\mathrm{S}_{\alpha}
\end{array}
$$

\subsection{Mesh Generation and Grid independence study}

Mesh generation was performed using ICEM-CFD software. In this software different grid generation techniques are available for producing different elements in computational domain like tri, quad in 2D and tetra, prism, hex in $3 \mathrm{D}$. In current study, for creating only hex 
Vol. 4, Issue 1, January 2017

elements in computational domain blocking technique was used. Hex element domain was used because these elements have best quality and best quality computational results are obtained and also cell count is reduced in large extent.

Grid independence study was carried out to find out the optimum grid size without compromising the accuracy of results. Different mesh sizes were tested in order to examine the effect of number of cell volumes on the Nusselt number as shown in Fig. 2

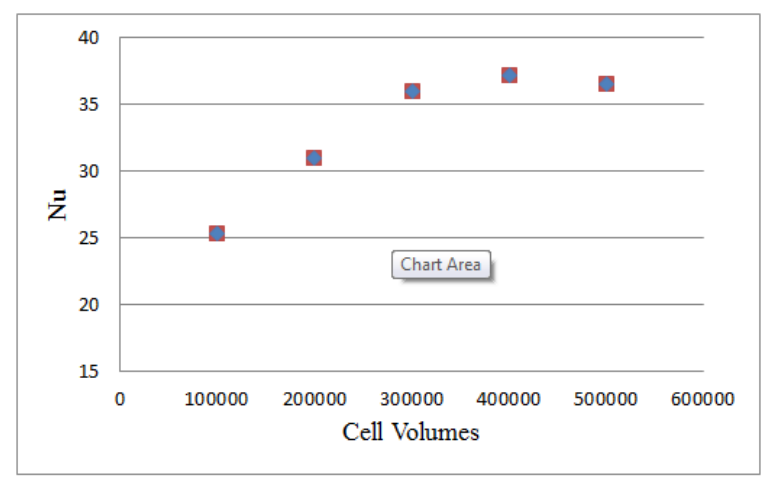

Fig. 2 Grid Independence study

It can be observed that the Nusselt number for water increases linearly till an optimum number of cell volumes is reached. Beyond this, any further increase in the number of cell volumes only increases the computational time, without any significant improvement in the Nusselt number. Similar trend was also observed with the nanofluids. So this "optimum" mesh size was selected for further study with both water and the nanofluids.

\subsection{Numerical Solution Strategy}

The commercial CFD solver FLUENT 13.0 was used to perform the simulations, based on finite volume approach to solve the governing equations with a segregated solver. The first-order upwind scheme was used for discretization of convection terms, volume fraction, energy, turbulent kinetic and turbulent dissipation energy. The SIMPLE algorithm was used to resolve the coupling between velocity and pressure fields. The convergence criterion is based on the residual value of calculated variables such as mass, velocity components, turbulent kinetic $(\mathrm{k})$, turbulent dissipation energies $(\varepsilon)$, and energy and volume fraction. In the present calculations, the initial residual values were set to 10-3 for all variables, except for energy for which 10-6 is used. The under-relaxation factors used for the stability of the converged solutions are set at their default values. The numerical simulation was decided as converged when the sum of normalized residuals for each conservation equation and variables was less than the set residual values. However, the residual for the continuity equation reached a minimum plateau before the value of 10-4, thus additionally, the mass balance and temperature outlet are monitored on the flux report and was used as a secondary indicator of convergence when the net imbalance is less than $1 \%$ of the inlet flux through the domain boundary.

\subsection{Post Processing}

4.4.1 Velocity Vector Plot

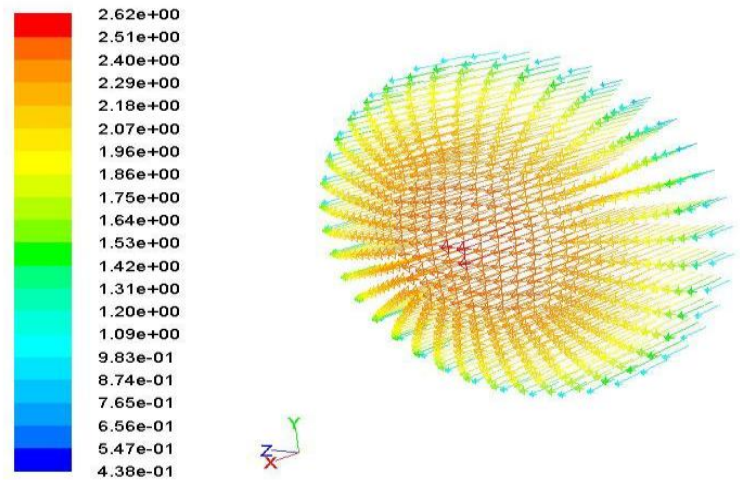

Fig. 3 Velocity vector plot at vertical cross section

Fig. 3 shows that, velocity vector plot at horizontal and vertical cross section at Reynolds number 10000 respectively. It can be seen from both the figure the velocity at near the wall is less than the velocity at Centre of the tube, so the velocity increases from wall region to middle region of tube.

\subsubsection{Temperature Plot}

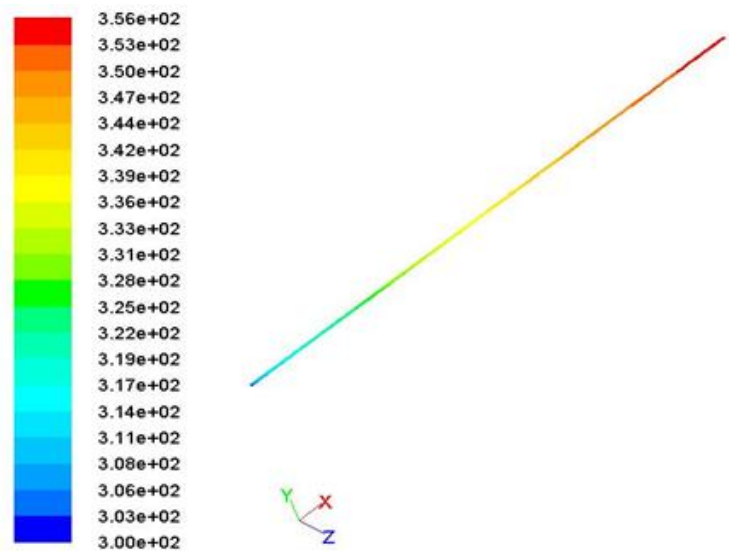

Fig. 4 Temperature plot for water

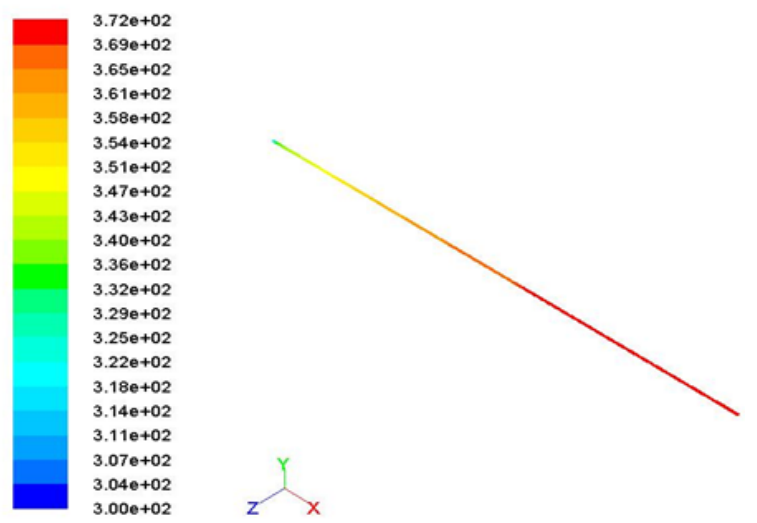

Fig. 5 Temperature plot for Nanofluid 
Vol. 4, Issue 1, January 2017

Fig. 4 and 5 shows the temperature plots for the water and In nanofluid with $0.3 \%$ concentration, the average relative nanofluid at Reynolds number 10000 respectively. From error between experimental data and CFD results based on the colour map blue to red, ranges from 300-356 for water single-phase model is $15.61 \%$ while for two-phase model and for nanofluid it is $300-372$ although both the fluid is $10.67 \%$. Also for nanofluid with $1 \%$ concentration, the heated with same constant heating temperature. Hence it is average relative errors for single-phase and two phase clear that heat transfer in nanofluid is more than water.

\section{RESULT AND DISCUSSION} models are $13.24 \%$ and $3.17 \%$ respectively, So two phase model can be used for prediction of heat transfer coefficient of nanofluid.

Fig. 6 and 7 shows that the heat transfer coefficient of $\mathrm{Al}_{2} \mathrm{O}_{3} /$ Water nanofluid with $0.3 \%$ and $1 \%$ volume fraction versus Reynolds number under two phase and single phase models. It can be seen that at the constant Reynolds number heat transfer coefficient increases with nanoparticle volume fraction. Also it is clear from this figure that the two-phase model is more precise than single-phase model.

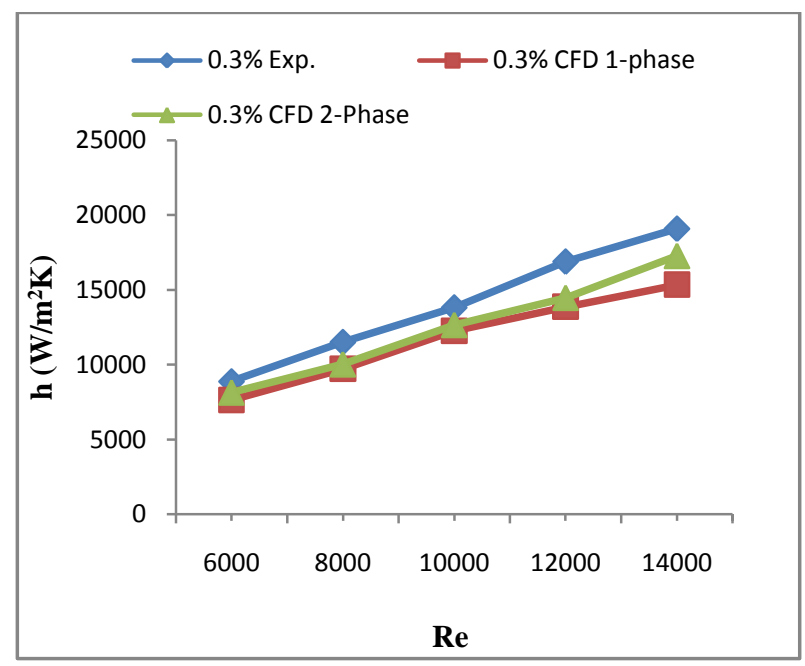

Fig. 6 Comparison between CFD predictions (based on single-phase and two-phase models) and experimental data in $\mathrm{Al}_{2} \mathrm{O}_{3}$ /Water nanofluid with $0.3 \%$ volume fraction and surface temperature of $50^{\circ} \mathrm{C}$

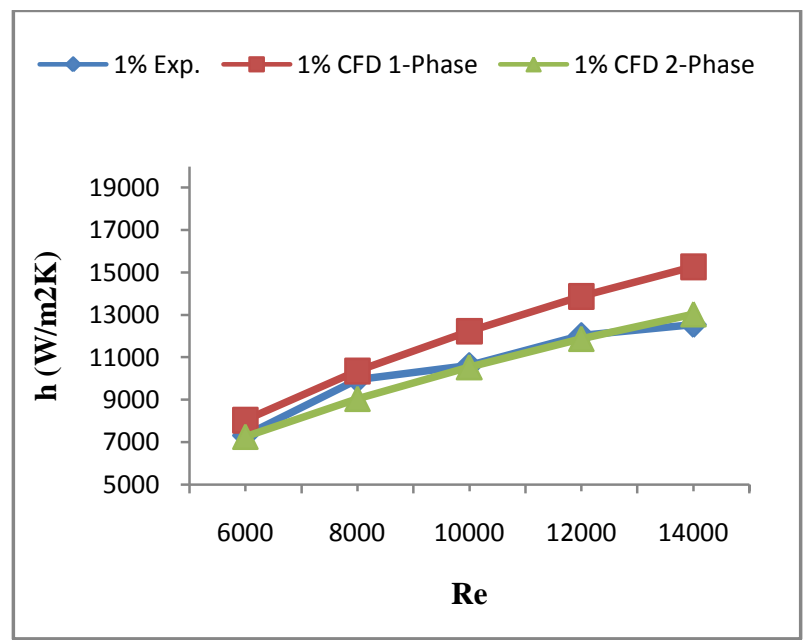

Fig. 7 Comparison between CFD predictions (based on single-phase and two-phase models) and experimental data in $\mathrm{Al}_{2} \mathrm{O}_{3} /$ Water nanofluid with $1 \%$ volume fraction and surface temperature of $60^{\circ} \mathrm{C}$.
Fig. 8 shows the experimental and CFD prediction heat transfer coefficients for four kinds of nanofluids at different concentrations $(0.3 \%, 0.6 \%, 0.8 \%$ and $1 \%)$ versus Reynolds number. There is a good agreement between the experimental data and CFD results. It can be found that the heat transfer coefficient for nanofluids increases with increasing particle volume fraction.

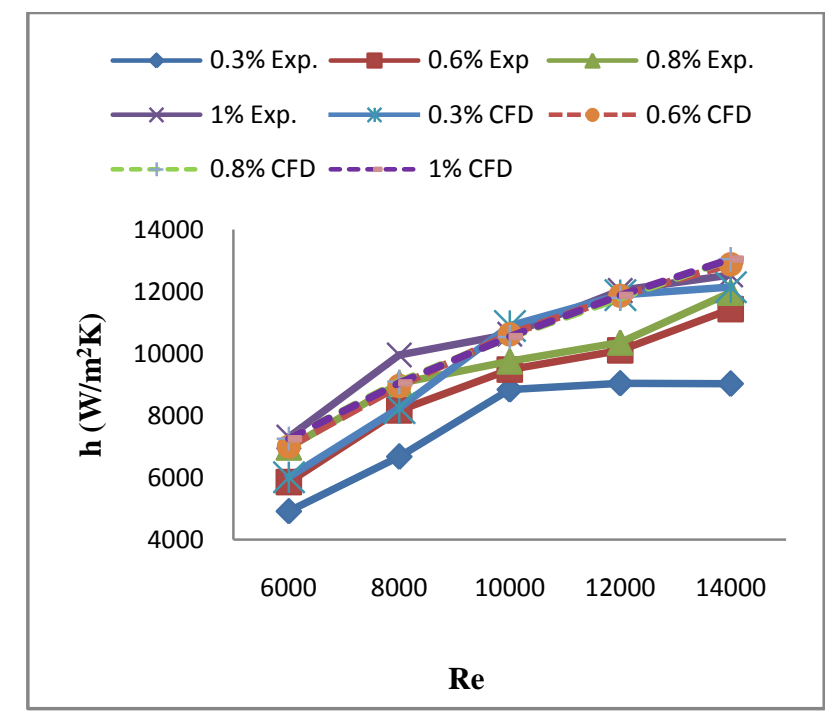

Fig. 8 Experimental and CFD prediction of heat transfer coefficient for $\mathrm{Al}_{2} \mathrm{O}_{3} /$ Water nanofluid at different concentrations versus Reynolds number.

\section{VI.CONCLUSION}

In the present work CFD models have been developed to predict the convective heat transfer coefficient of $\mathrm{Al}_{2} \mathrm{O}_{3} /$ water nanofluid with different volume fraction in circular tube by using experimental data. The volumeaveraged continuity, momentum and energy equations were numerically solved by using ANSYS FLUENT version 13.0. Single phase and two phase models have been used for prediction of heat transfer coefficient and Nusselt number. Some of the concluding remarks from the study are as follows,

1. At constant Reynolds number convective heat transfer coefficient for nanofluid is higher than that of the base fluid.

2. Heat transfer coefficient is found to be enhanced with increasing the nanoparticle concentration and Reynolds number

3. Two phase model of nanofluid is more precise than the single phase model for prediction of heat transfer coefficient and Nusselt number. 
Vol. 4, Issue 1, January 2017

4. Computed heat transfer coefficients and Nusselt domain Nanofluid and Computational Fluid Dynamics. numbers of nanofluid with different volume He has published five papers in international conference concentrations are in good agreement with theoretical and two in national conference and attended many correlations and the experimental data.

At fixed Reynolds number 6000, the heat transfer coefficient for $1 \% \mathrm{Al}_{2} \mathrm{O}_{3}$ /water nanofluid increases by 1.55 times over the base fluid which indicates that the use of nanofluid can significantly increase the heat transfer capabilities of cooling systems.

\section{REFERENCES}

[1] M. HaghshenasFard, M. Nasr Esfahany, M.R. Talaie, "Numerical study of convective heat transfer of nanofluids in a circular tube two-phase model versus single-phase model", International Communications in Heat and Mass Transfer, 37 (2010) 91-97.

[2] M. Akbari, N. Galanis, A. Behzadmehr, "Comparative analysis of single and two-phase models for CFD studies of nanofluid heat transfer", International Journal of Thermal Sciences, 50 (2011) $1343 \mathrm{e} 1354$.

[3] MostafaKeshavarzMoraveji, Mehdi Darabi, Seyyed Mohammad Hossein Haddad, Reza Davarnejad, "Modeling of convective heat transfer of a nanofluid in the developing region of tube flow with computational fluid dynamics", International Communications in Heat and Mass Transfer, (2011).

[4] Perumal Kumar, RajamohanGanesan, "A CFD Study of Turbulent Convective Heat Transfer Enhancement in Circular Pipeflow", International Journal of Civil and Environmental Engineering, 62012.

[5] P.Kumar, "A CFD Study of Heat Transfer Enhancement in Pipe Flow with $\mathrm{Al}_{2} \mathrm{O}_{3}$ Nanofluid", World Academy of Science, Engineering and Technology, 572011

[6] S. P. Pendalwar, U.V. Bhandarkar and S. V. Prabhu, "Experimental and numerical investigation on convective heat transfer coefficient for the turbulent flow through pipe using nanofluid", Internantional Journal of Micro and Nano System, 1(1), 2009, pp.9-14.

[7] S.M. Fotukian, M. Nasr Esfahany, "Experimental investigation of turbulent convective heat transfer of dilute $\gamma-\mathrm{Al}_{2} \mathrm{O}_{3} /$ water nanofluid inside a circular tube", International Journal of Heat and Fluid Flow 31 (2010) 606-612.

[8] R. MokhtariMoghari , A. Akbarinia , M. Shariat, F. Talebi , R. Laur, "Two phase mixed convection $\mathrm{Al}_{2} \mathrm{O}_{3}$-water nanofluid flow in an annulus", International Journal of Multiphase Flow 37 (2011) 585-595.

[9] F. W. Dittus, L. M. K. Boelter, "Heat transfer in Automobile Radiators of Tubular Type." University of Califirnia Press, Berkeley, CA, 1930, pp $13-18$.

[10] Seider, E. N., Tate, G.E., "Heat transfer and pressure drop of liquid in tubes", Industrial Engineering Chemistry 28(12), 1429 - 1435.

[11] Maiga. S.E.B., Nguyen, C.T., Galanis,N., Roy. G., Mare. T., "Heat transfer enhancement in turbulent tube flow using $\mathrm{Al}_{2} \mathrm{O}_{3}$ nanoparticles suspension." International Journal of Numerical methods for Heat and Fluid Flow 16(3), 275 - 292

[12] SartikK.Das, Stephen U. S. Choi, Wenhua Yu, T. Pradeep, "Nanofluid Science and Technology", 2007, USA

\section{BIOGRAPHY}

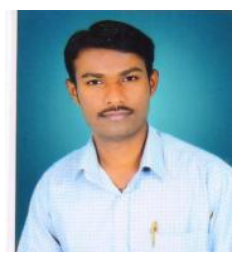

Prof. Pankaj s. Ghatage M. Tech (CAD/ CAM/CAE), B.E.(Mechanical). Mr. Pankaj Ghatage is working as Assistant Professor at Automobile Engineering Department, Rajarambapu Institute of Technology (RIT), Islampur. An Autonomous Institute Affiliated to Shivaji University Kolhapur (India). He has four years of experience in teaching. He is working in the

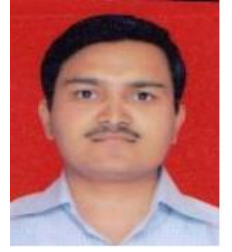

Dr. Abhijeet P. Shah Ph. D. (Mechanical) M.E. (Mech-Design), B.E. (Automobile), Dr. Abhijeet Shah is working as Assistant Professor at Automobile Engineering Department, Rajarambapu Institute of Technology (RIT), Islampur. An Autonomous Institute Affiliated to Shivaji University Kolhapur (India). He has thirteen years of experience in teaching. His areas of research include heat transfer and CFD. He has published seven papers in international journals and eleven papers in national and international conferences. 